\title{
Basilar impression complicating osteogenesis imperfecta type IV: the clinical and neuroradiological findings in four cases
}

\author{
M Hayes, G Parker, J Ell, D Sillence
}

\begin{abstract}
Objectives-To describe the clinical and neuroradiological features of basilar impression in patients with osteogenesis imperfecta type IV.

Methods-Four patients with basilar impression were ascertained in a population study of osteogenesis imperfecta. All four had detailed clinical and neuroradiological examination with both CT and MRI of the craniocervical junction andposterior fossa structures.

Results-All four showed significant compression of the posterior fossa structures and surgical decompression was performed with relief of symptoms.

Conclusion-Symptoms of cough headache and trigeminal neuralgia occurring in patients with osteogenesis imperfecta are indications for detailed clinical and neuroradiological investigation to document basilar impression.
\end{abstract}

(F Neurol Neurosurg Psychiatry 1999;66:357-364)

Keywords: basilar impression; osteogenesis imperfecta

Neurology, Concor

Hospital, Concord

NSW, Australia

$M$ Hayes

Department of Radiology, Royal

Prince Alfred Hospital, Camperdown, NSW, Australia

G Parker

Department of Neurology, Royal Prince Alfred Hospital, Camperdown, NSW, Australia

J Ell

University

Department of

Paediatrics and Child

Health, Royal

Alexandra Hospital for

Children, Parramatta,

NSW, Australia

D Sillence

Correspondence to: Professor D Sillence, Department of Clinica Genetics, Royal Alexandra Hospital for Children, PO Box 3515, Parramatta, NSW 2124, Australia. Telephone 006129845 3273; fax 0061 298453204 .

Received 2 March 1998 and in revised form

3 June 1998

Accepted 8 July 1998 severe neurological consequences. Most commonly this condition is due to a congenital developmental anomaly, but it may also occur secondarily to a generalised bone disease such as osteomalacia, Paget's disease, hyperparathyroidism, rheumatoid arthritis, and osteogenesis imperfecta.

Osteogenesis imperfecta is a clinically and genetically heterogeneous group of heritable disorders of connective tissue characterised by reduced bone mass (osteopenia) with associated bone fragility. ${ }^{4}$ Skeletal manifestations are due to a generalised deficiency of development of both membranous and endochondral bone and include markedly thinned calvarium with delayed closure of fontanelles and sutures and excessive Wormian bone formation. Until recently, the reported association of osteogenesis imperfecta and basilar impression was considered rare. Furthermore, it was presumed that basilar impression occurs equally in all types of osteogenesis imperfecta. However, the combination of increased awareness coupled with the advent of improved neuroimaging has led to wider screening and detection of the condition. $^{5}$ This might have remained an academic exercise were it not for anaesthetic and neurosurgical advances in foramen magnum and posterior fossa decompressive surgery which reduce the morbidity and mortality from intervention. $^{36}$

Screening of at risk people is likely to disclose a proportion with subclinical basilar impression. Closer surveillance can then be instituted. However, a craniocervical malformation is likely to be clinically suspected before neuroimaging is performed. The range of clinical symptoms is determined by the particular combination of direct compression and vascular disruption resulting from upward displacement of the floor of the posterior fossa (table 1). Some of these symptoms may be transient, progressive, or catastrophic. The specific structures that may be involved include the upper cervical cord, medulla, pons, mid-brain, cerebellum (medial), and the vertebrobasilar system.

The close proximity of the structures implies that only a very minor variation in displacement between patients can give rise to a wide variety of symptoms. Additionally, only minor degrees of progression, either gradual or sudden (due to trauma), may have major clinical consequences. This paper discusses the clinical presentation of four cases of basilar impression in osteogenesis imperfecta correlated with the anatomical abnormalities as defined by MRI. The following definitions have been employed for the purpose of analysis. ${ }^{7}$

\section{BASILAR INVAGINATION}

This is defined ${ }^{1}$ as when the tip of the dens projects more than $5 \mathrm{~mm}$ above a line joining the hard palate to the posterior lip of the foramen magnum (Chamberlain's line) or the tip of the dens is $>7 \mathrm{~mm}$ above McGregor's line (the back of the hard palate to the lowest point of the occipital squama) (fig 1A).

BASILAR IMPRESSION

This is defined as when there is elevation of the floor of the posterior fossa including occipital

Table 1 Neurological symptoms resulting from basilar impression

Headache: characteristically occipital and precipitated by cough and laughter.

Trigeminal neuralgia

Weakness in legs

Weakness in arms

Sleep apnoea

Seizures
Vertigo or imbalance precipitated by head movement 
condyles and foramen magnum. In coronal views the atlanto-occipital joints are normally below a line joining the digastric notches (fig 1B). In sagittal views it can be confirmed by the Klaus height index (height of the posterior fossa), ${ }^{1}$ the line drawn perpendicular between Twining's line (fig 5A), and the tip of the odontoid process (average $=40-42 \mathrm{~mm}$ ). This dimension is abnormal when $<30 \mathrm{~mm}$. Twining's line is the line drawn from the tuberculum to the internal occipital protuberance. Where the tip of the dens cannot be visualised, a modification is the distance between Twining's line and McCrae's line (average $40 \mathrm{~mm}$ ) which is definitely abnormal when $<30 \mathrm{~mm}$. McCrae's line is drawn from the middle of the anterior lip of the foramen magnum to the posterior lip of the foramen magnum.

Although basilar invagination (particularly as defined by McGregor's line) is usually present in patients with basilar impression, the converse is not necessarily true and basilar invagination may be present without basilar impression.

\section{Case reports}

CASE 1

An 18 year old man with osteogenesis imperfecta type IV B was diagnosed with osteogenesis imperfecta at birth after the detection of multiple fractures. He weighed $2.15 \mathrm{~kg}$. Dislocation of an ankle joint and bilateral inguinal hernias were present. There was no family history of osteogenesis imperfecta. He has had a total of 20 fractures and intramedullary rodding of both femurs on three occasions. He was noted to have white sclerae, dentinogenesis imperfecta and normal hearing.

At the age of 8 years, he had intermittent dizziness. When reviewed at the age of 9 years, he was asymptomatic and no neurological signs were elicited. Head circumference was $55 \mathrm{~cm}$ (>98th centile), and height $100 \mathrm{~cm}$ (<third centile). Both frontal bossing and occipital overhang were present. He had a barrel chest but no significant kyphoscoliosis. Three years later (aged 12 years) he complained of brief episodes of acute occipital headache brought on by laughing or coughing. He also had intermittent vertigo precipitated by neck movements, particularly extension.

Examination now disclosed increased tendon reflex of the right knee and ankle but with flexor plantar responses. Downbeat nystagmus was present. Within 9 months he had developed bilateral extensor plantar responses. Functionally, he required a wheelchair but could stand to transfer.

Basilar impression was diagnosed on skull radiography (fig 2A). An MRI showed compression of the cerebellar hemispheres and pons by the elevated bony structures of the floor of the posterior fossa and C1. The medulla was elongated and there was dilatation of the lateral and third ventricles (fig 2B-E).

A year later, a posterior-cervical fusion was performed to stabilise the craniocervical region before transoral odontoidectomy (aged 15 years). A preoperative MRI indicated further upbending of the clivus. Examination now disclosed generalised hyperreflexia in the lower limbs with unsustained bilateral clonus. A clivectomy was performed via a transoral approach as previously described. ${ }^{6}$ Twelve months after clivectomy he had mild persistence of palatopharyngeal incompetence. Plastic surgical repair of his palate was successfully undertaken with improved function. When reviewed 7 years after clivectomy he had no symptoms of basilar impression and neurological examination was normal. He remains a wheelchair user but has continued to improve postoperatively and is pursuing university studies.

\section{CASE 2}

A 20 year old man with osteogenesis imperfecta type IV B was diagnosed at birth after the detection of a dislocated ankle and bowed legs. $\mathrm{He}$ weighed $2.33 \mathrm{~kg}$. Three hernia operations were performed in infancy. There was no family history of osteogenesis imperfecta and he subsequently had an unaffected sister. He had femoral fractures at the age of 12 and 15 months and has had a total of about 15 fractures.

When assessed at the age of 8 years, he was less than the third centile for height and weight

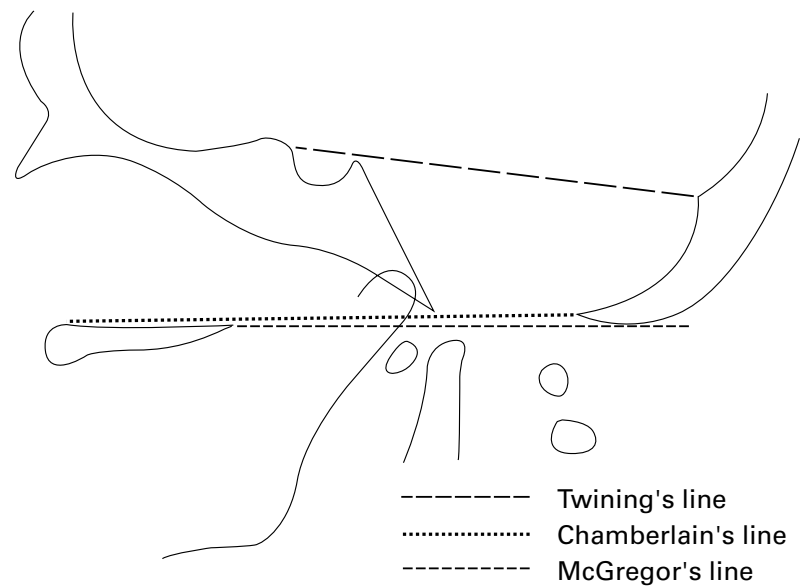

B

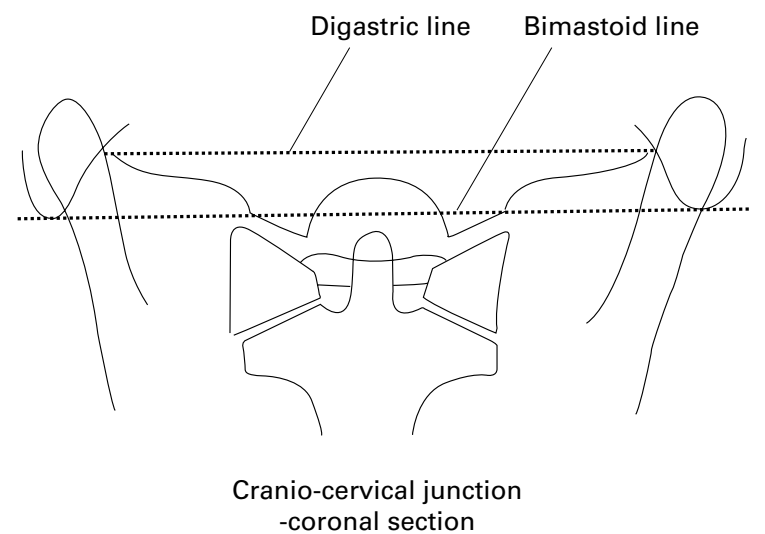

Figure 1 (A) Diagrammatic representation of the osseous landmarks in a sagittal reconstruction of the craniocervical junction. (B) Diagrammatic representation of osseous landmarks in a coronal reconstruction of the craniocervical junction. 

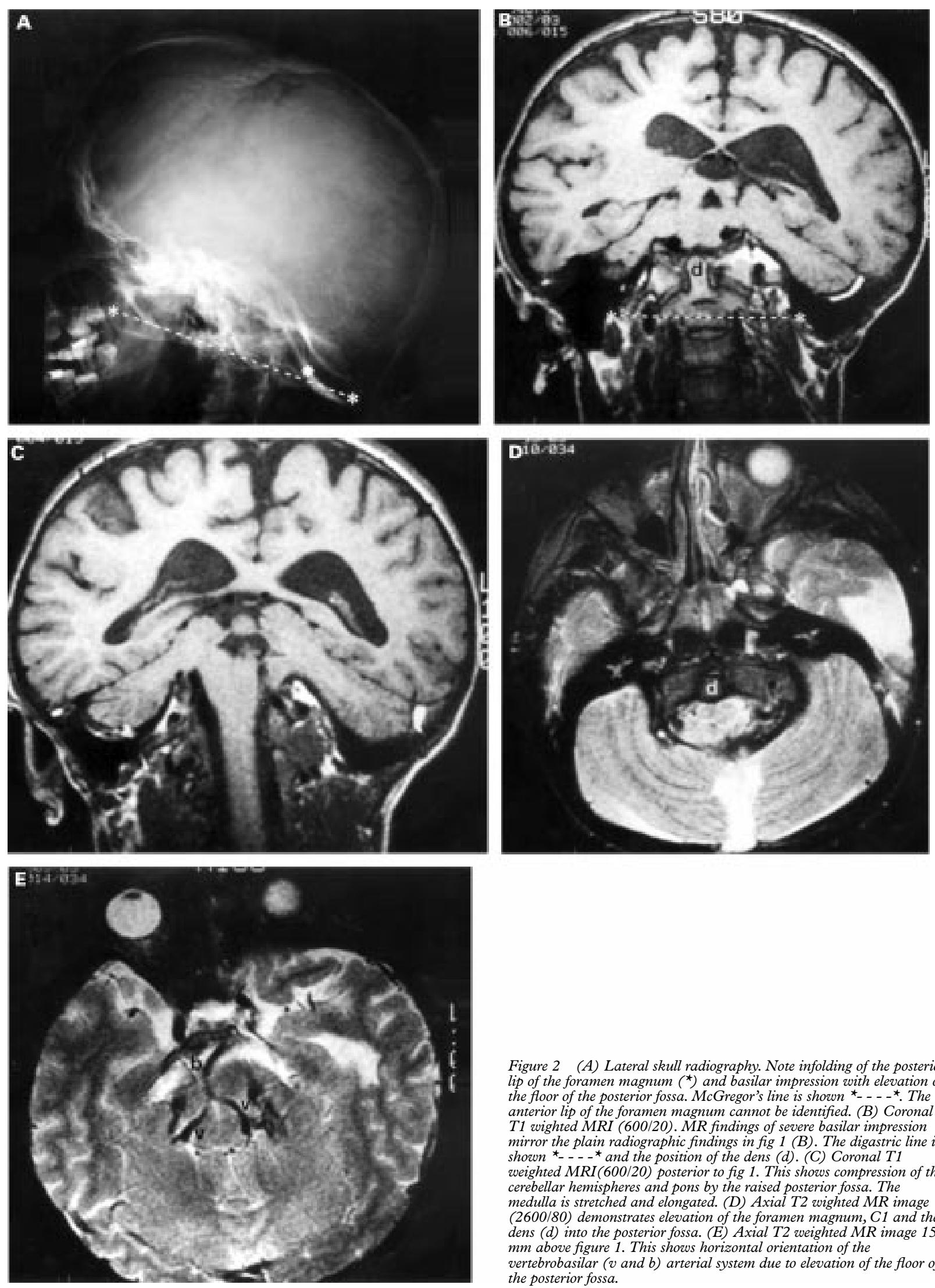

Figure 2 (A) Lateral skull radiography. Note infolding of the posterior lip of the foramen magnum $\left(^{\star}\right)$ and basilar impression with elevation of the floor of the posterior fossa. McGregor's line is shown * . - * . The anterior lip of the foramen magnum cannot be identified. (B) Coronal T1 wighted MRI (600/20). MR findings of severe basilar impression mirror the plain radiographic findings in fig 1 (B). The digastric line is shown * . . - and the position of the dens (d). (C) Coronal T1 weighted MRI(600/20) posterior to fig 1. This shows compression of the cerebellar hemispheres and pons by the raised posterior fossa. The medulla is stretched and elongated. (D) Axial T2 wighted MR image (2600/80) demonstrates elevation of the foramen magnum, C1 and the dens (d) into the posterior fossa. (E) Axial T2 weighted MR image 15 $\mathrm{mm}$ above figure 1. This shows horizontal orientation of the

vertebrobasilar $(v$ and $b$ ) arterial system due to elevation of the floor of the posterior fossa.

$(13.5 \mathrm{~kg})$. Head circumference was $54 \mathrm{~cm}$ dentinogenesis imperfecta was evident. He (50th-98th centile) and he exhibited frontal and occipital bossing. He was barrel chested but there was no evidence of kyphoscoliosis. His sclerae and hearing were normal but required crutches to walk.

He remained quite well until the age of 16 years. At this time he developed cough headache in the left occipital area associated 
with brief (seconds) sensations of postural instability and presyncope. These symptoms were more prominent on awakening and early in the day. He also described occasional difficulty with fixation and focusing on objects. An isolated generalised clonic-tonic seizure occurred and he was started on $200 \mathrm{mg}$ carbamazepine twice daily.

At the age of 17 years he developed trigeminal neuralgia in the second and third divisions, while still on carbamazepine. Chewing and talking seemed to exacerbate the episodes but there were no trigger points on the face. The pain was often severe enough to wake him from sleep. Examination disclosed marked occipital overhang and bilateral horizontal nystagmus but no other cranial nerve abnormality. The deep tendon reflexes were increased, particularly in the lower limbs, with non-sustained patellar clonus. The plantar responses were flexor and sensory examination was normal. A $\mathrm{CT}$ of the craniocervical region (fig 3A, B, C) disclosed basilar impression with shortening and flattening of the clivus and impression of the margins of the foramen magnum and $\mathrm{C} 1$ and $\mathrm{C} 2$ into the posterior fossa.
Brain MRI (fig 3D) showed an abnormal clivus with severe angulation of the brainstem and compression of the posterior cerebral vessels against the brainstem. It was hypothesised that the trigeminal neuralgia was related to compression of the nerve root on the left by a branch of the basilar artery. The trigeminal neuralgia gradually worsened and was not controlled by $750 \mathrm{mg}$ carbamazepine daily. After being symptomatic for 18 months, examination now disclosed first degree upbeat nystagmus in addition to the bilateral horizontal gaze sporadic nystagmus. Coordination was preserved but the plantar responses were now extensor bilaterally. He proceeded to surgery 3 months later (aged 19 years).

Transoral removal of the anterior arch of C2, the tip of the odontoid, and a clivectomy were performed. The craniocervical junction was stabilised with a Ransford loop supporting the occiput and fixed at its lower end by tieing it to the upper ribs. Within a month of surgery there was a total remission of the trigeminal neuralgia.. He has persistent up beat nystagmus. He had recrudescence of the trigeminal neuralgia 2.5 years after clivectomy but the
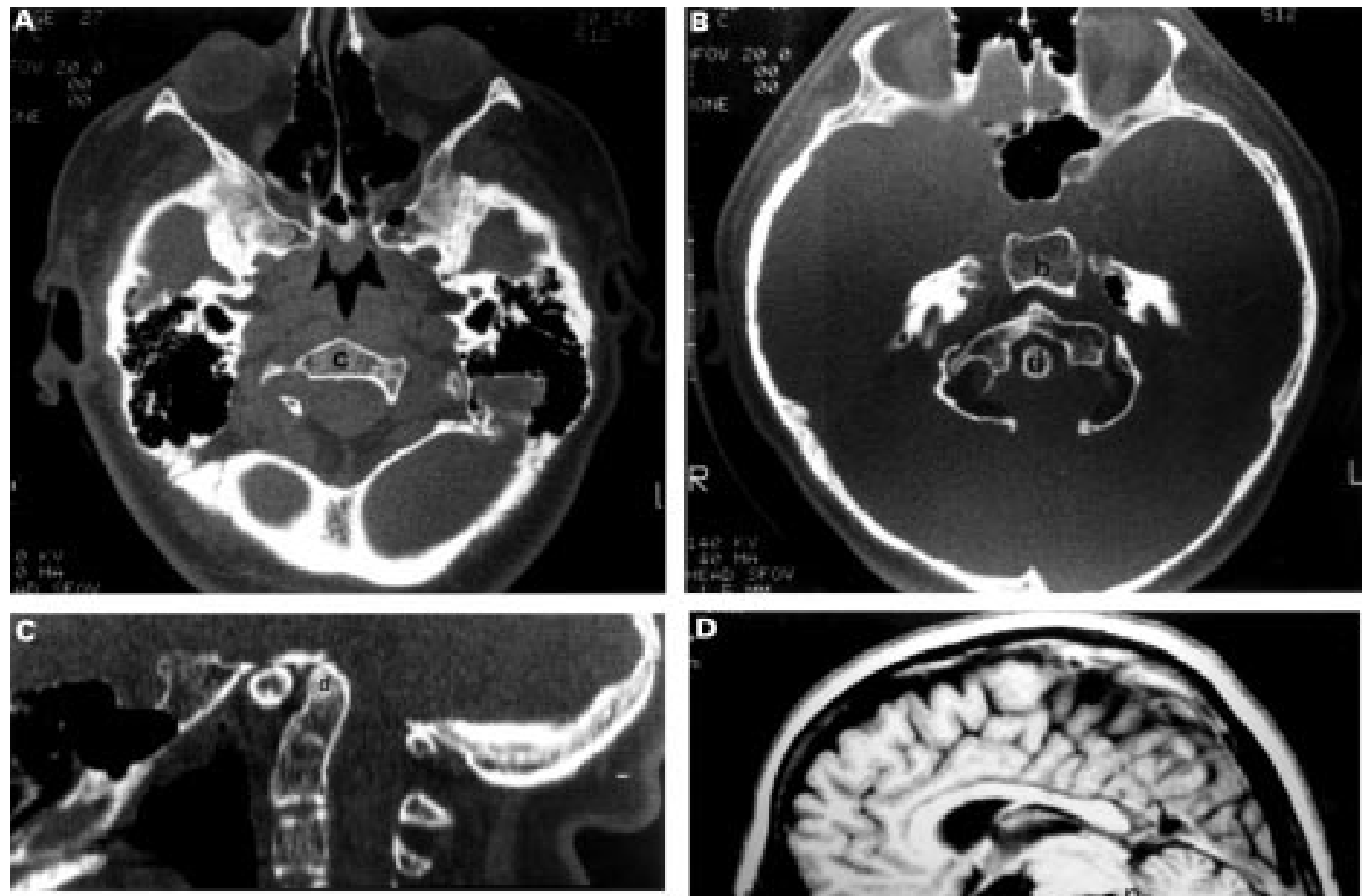

Figure 3 (A) Axial CT at the skull base showing infolding of the floor of the posterior fossa. The body of the axis (c) is located above the skull base. (B) Axial CT of the level of the petrous apices showing the dens (d) and C1 vertebral body at this level. The basiocciput (b) is hypoplastic. (C) Sagittal reformation of CT images confirms severe basilar impression. The dens (d) and C1 are located at the level of the anterior foramen magnum. The clivus (b) is horizontal. (D) Sagittal T1 weighted MRI shows severe basilar impression with hypoplasia of the basiocciput (b) and compression of the pontomedullary junction (p) by the elevated dens. A Chiari malformation is also present with inferior displacement of the cerebellar tonsils $(t)$. This may be due to unfolding of the posterior lip of foramen magnum. A small syrinx is also noted at the C4 level.

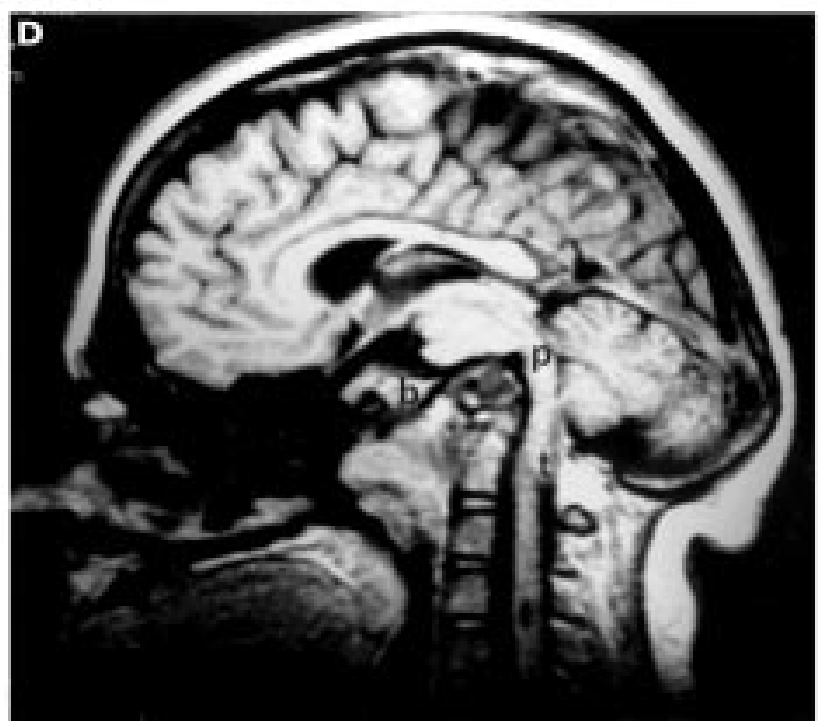


neuralgia was intermittent and more easily controlled with carbamazepine or analgesic medication. He was otherwise well and has remained stable 6.5 years after clivectomy.

CASE 3

Unlike the previous two cases, this 32 year old man with osteogenesis imperfecta type IV B remained undiagnosed until he presented with symptoms and signs of basilar impression at the age of 30 years. He had a normal birth (weight $4.1 \mathrm{~kg}$ ) and was the eighth of nine children in a family without a history of osteogenesis imperfecta. As a child aged 5 years, he was inconclusively investigated for short stature. His first fracture was at the age of 14 and subsequently he has had five or six further fractures. Examination at the age of 30 disclosed a man with short stature, $146.5 \mathrm{~cm}$ (<third centile), head circumference of 59.5 $\mathrm{cm}$ and temporal and occipital bulging. He had normal sclerae and hearing and was barrel chested. Dentinogenesis imperfecta was present.

He presented with a 6 month history of a progressively unsteady, wide based gait. $\mathrm{He}$ experienced difficulty walking downstairs and complained of stiffness in his lower limbs accompanied by some numbness, more marked in the right leg. He noticed that coughing elicited pain at the back of his head and that neck extension produced some vertigo. He had no difficulty with speech or swallowing.

Examination disclosed bilateral horizontal gaze nystagmus without a vertical component. Apart from mild hyperreflexia, the upper limbs were normal. However, the lower limbs were markedly hypertonic with brisk reflexes and sustained ankle clonus. The plantar responses were flexor. The gait was spastic and grossly ataxic. Heel-shin testing was moderately incoordinate. $\mathrm{He}$ also exhibited some sensory abnormality, loss of vibration sense to the knees, minor loss of proprioception at the toes bilaterally, and some asymmetric pain and temperature loss below the knees.
Skull radiography showed evidence of basilar impression as well as multiple Wormian bones in the lambdoid suture. Brain MRI (fig 4A) showed gross platybasia and basilar impression producing compression of the lower pons and medulla with an associated focal cyst in the upper cervical cord. There was no evidence of hydrocephalus or Arnold-Chiari malformation. Craniocervical CT (fig 4B) showed the arch of $\mathrm{C} 1$ and dens projecting into the posterior fossa almost as high as the apex of the petrous temporal bones.

$\mathrm{He}$ was referred for surgery involving transoral odontoidectomy, clivectomy, and subsequent posterior occipital fusion. Postoperatively he improved steadily with a return to walking although somewhat broad based. The nystagmus has resolved. Just as in patients 1 and 2 he has mild palatopharyngeal incompetence which also improved over the first year after operation. Six and a half years postoperatively he has a slightly spastic gait and decreased proprioception. He has just completed a graduate diploma in electrical engineering.

CASE 4

This 38 year old woman with osteogenesis imperfecta (type IVA) was a breech delivery (weight $2.3 \mathrm{~kg}$ ) but no abnormalities were detected. Subsequently she had seven childhood fractures, the first (clavicle) at the age of 15 months. A brother also has osteogenesis imperfecta type IVA.

She was involved in sports and dancing until the age of 14 years when she noticed that these activities precipitated a throbbing headache that was either diffuse or localised to the occipital area. She was also aware of some dizziness and loss of balance. These symptoms developed with coughing, sneezing, laughing, and bending over. Straining to lift heavy objects and even getting out of bed in the morning became troublesome. Severe symptoms would subside within minutes if she sat
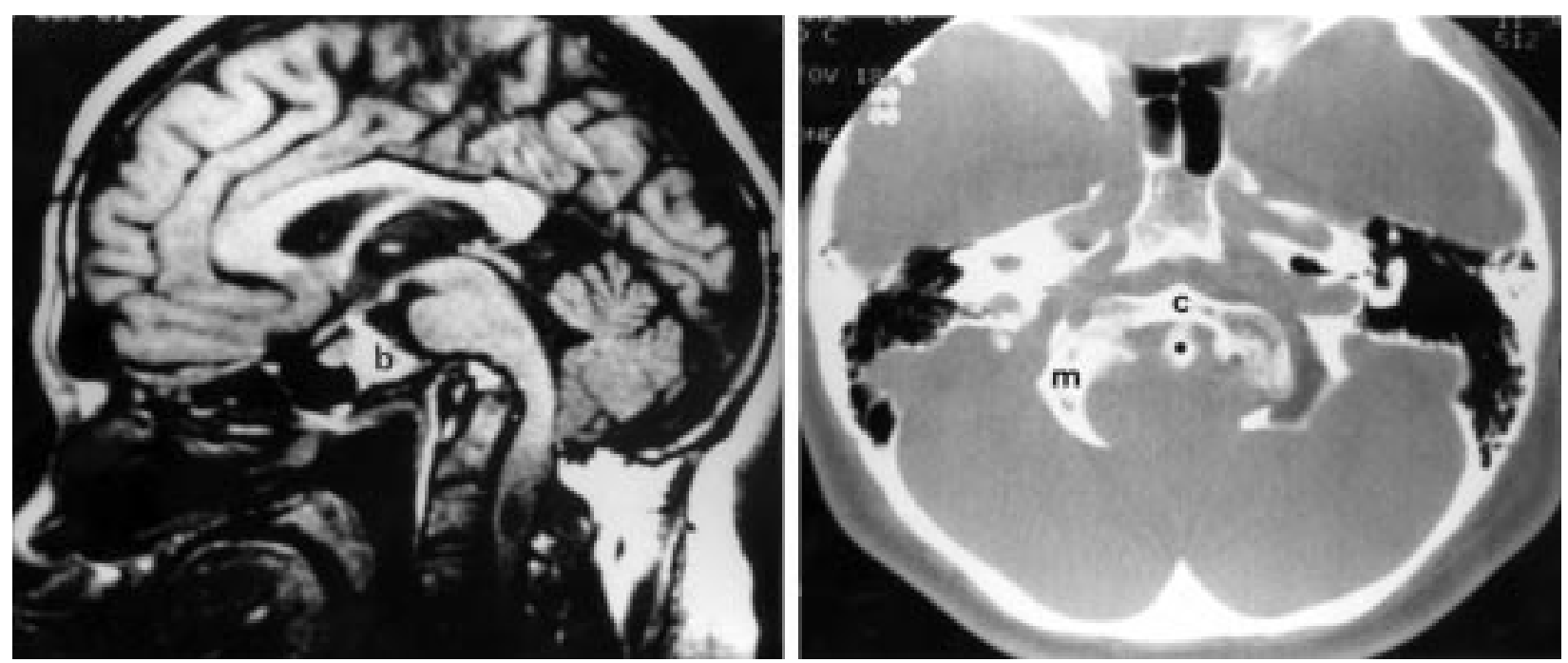

Figure 4 (A) Sagittal T1 weighted (600/20) MRI shows deformity of the pons and medulla due to basilar impression. Note basiocciput hypoplasia (b). There is an associated intramedullary cyst in the cervical spine at the C3 level. There is no Chiari malformation but kinking of the upper cervical cord is present. (B) Axial CT of the level of the mid-petrous temporal bone showing a portion of the margin of foramen magnum ( $m$ ), the anterior arch of C1 (c), and the dens $\left({ }^{\star}\right)$ displaced into the posterior fossa due to basilar impression. 

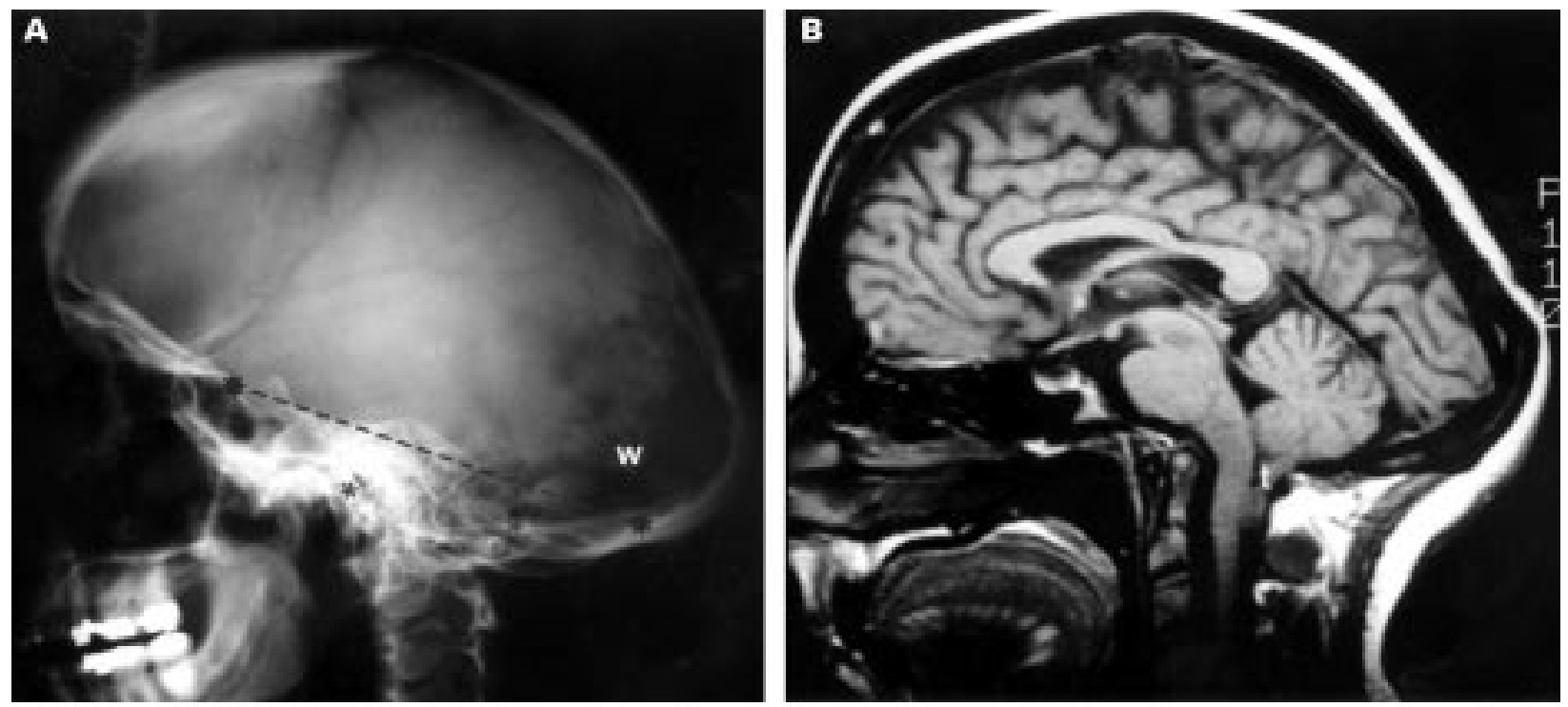

Figure 5 (A) Lateral skull radiography shows typical elevation of the floor of the posterior fossa seen in basilar impression. Note Wormian bones (W). The foramen magnum is elevated (between ${ }^{\star}$ ) and the posterior fossa is shallow. Twining's line is shown * - - *. (B) Sagittal T1 weighted MRI shows moderate basilar impression with a Chiari malformation. The cerebellar tonsils $(t)$ protrude inferiorly to the level of the posterior arch of $C 2$. The degree of pontine compression is less marked than in cases (1-3). (C) Axial T1 weighted image through the foramen magnum shows medullary compression $(m)$ by the herniated tonsils and elevation of the floor of the posterior fossa.

or stood perfectly still, usually holding her head or being supported by a chair or a wall.

At the age of 26 she was referred to a neurologist for cough headache. She was noted to have a short neck and unusual skull shape, but the remainder of the examination was normal. Skull radiography and CT showed marked basilar impression with a normal ventricular system. Over the ensuing 10 years her symptoms remained well controlled by leading a careful and sedate lifestyle; only coughing remained a frequent problem. Unsteadiness or imbalance could be induced by turning quickly while walking, standing, or even lying in bed. These symptoms subsequently responded well to low dose clonazepam.

Examination at the age of 36 years disclosed a normal appearance apart from a prominent occiput, weight $55 \mathrm{~kg}$, sclerae mid-hue, and normal teeth. Gait was normal and there was no nystagmus. However, both knee reflexes were pathologically brisk. Repeat skull radiography disclosed basilar impression (fig 5A). Brain CT and MRI (fig 5B and $\mathrm{C}$ ) showed changes of basilar impression with compression of the upper medulla to a moderate degree only and Arnold-Chiari malformation. Over the next 12 months she had a progression in the severity of headache and deterioration of neurological signs in the lower limbs. A posterior decompression of the foramen magnum was performed with gradual amelioration of symptoms.

\section{Discussion}

Craniocervical disorders are not represented by a single set of symptoms and signs. As Schmidt et al pointed out, the neurological deficits encountered in patients with the same craniocervical malformation may be extremely varied, due to both the closely packed neuroana-

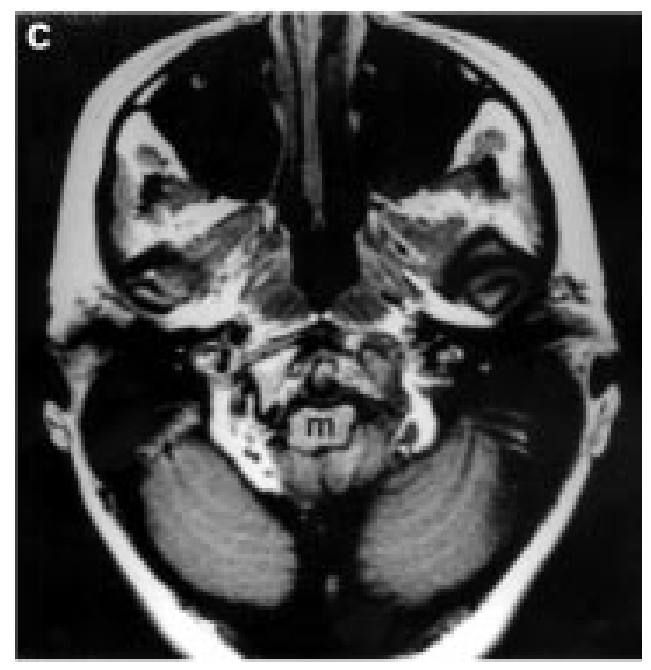

tomical structures and to the secondary effects of vascular and CSF disruption. ${ }^{1}$ The posterior cranial fossa, which contains the medulla, pons, part of the mid-brain, and the cerebellum is the largest and deepest of the cranial fossae. It is bounded anteriorly by the dorsum sellae, clivus of the sphenoid, and the basilar part of the occipital bone. The normally deep base of the fossa is formed by the remainder of the occipital bone, including the condylar portions aside the central foramen magnum. This concave structure forms a natural complement to the rounded convexity of the skull vault.

Virchow, in 1857, coined the term "platybasia" to describe an abnormal flattening of the base of skull, a defect which he attributed to abnormal bone development. Neurologically, this abnormality is not usually of any consequence. However, a more dramatic abnormality which Virchow (1876) termed basilar impression was occasionally seen in association with platybasia. In addition to flattening of the base of the skull, there was upward displacement (impression) of the basilar and condylar portions of the occipital bone. This resulted in an infolding or invagination of the foramen magnum, further diminution of the posterior fossa, 
Table 2 Neurological signs of compression of posterior fossa structures in basilar impression

Horizontal nystagmus Upbeat nystagmus

Downbeat nystagmus

Convergence nystagmus

Facial spasm

Nerve paresis

Pyramidal tract signs

Proprioceptive defect

Papilloedema in acute

hydrocephalus and consequent protrusion of the upper cervical spine into the anterior brainstem, with potentially disastrous neurological consequences.

Basilar impression is the commonest of the craniocervical malformations. Most cases are probably due to a congenital bone malformation of the posterior fossa (primary basilar impression), whereas secondary basilar impression such as in osteogenesis imperfecta, results from abnormally soft bone in effect slowly "melting" over the upright cervical spine, which progressively protrudes through the foramen magnum. Cerebellar tonsillar herniation (case 4) is a recognised association with both primary and secondary basilar impression. ${ }^{8}$ Conversely, basilar impression is found in about $40 \%$ of all adults treated for symptomatic Arnold-Chiari malformation. ${ }^{910}$

Although the onset of clinical symptoms can be sudden and precipitated by relatively minor trauma, the commonest course would seem to be a slowly progressive one as exemplified in all four cases. The most frequent symptom of basilar impression is headache and it is characteristically located in the neck and occipital region (table 1). ${ }^{1112}$ Although headache can occur spontaneously, it is typically triggered by movement and exercise as described in all the cases. Sometimes (case 4) the head is held rigid to minimise the discomfort. The headaches are often more severe in the morning (cases 2 and 4). Apart from precipitating headache, actions such as coughing, sneezing, and straining also induce giddiness or imbalance as described in all four cases. The mechanism of cough headache is unclear and some argue that it is not related to raised intracranial pressure. ${ }^{1314}$

Horizontal nystagmus is probably the commonest neurological sign elicited in symptomatic basilar impression (table 2). Bilateral horizontal gaze evoked nystagmus was seen in cases 2 and 3 . The fast phase is always in the direction of gaze. A marked bidirectionality to the nystagmus almost always implies a central cause, but is of relatively poor localising value occurring in both brainstem and cerebellar lesions. Drug toxicity needs to be excluded. Upbeat nystagmus developed in case 2 and this usually indicates structural disease in the brainstem, although precise localisation is unclear. ${ }^{15}$ Central otolithic pathways are implicated. Downbeat nystagmus (case 1) has the most precise localising value. This is strictly defined as nystagmus in the primary position with the fast phase in the downward direction. Contrary to Alexander's law (jerk nystagmus is maximal with gaze in the direction of the fast phase), it is often best seen with lateral and slightly downward gaze. ${ }^{16}$ The presence of downbeat nystagmus indicates an abnormality at the craniocervical junction and it is probably commoner in Arnold-Chiari malformations than basilar impression alone, as in this case. Downbeat nystagmus is not usually seen in brainstem pathology alone or in drug toxicity, although there are isolated case reports. Spinocerebellar degeneration needs to be excluded. Disruption of central vestibular pathways is the presumed mechanism.
Involvement of the lower cranial nerves has been described but seems to be relatively uncommon. ${ }^{12}{ }^{17}$ Facial nerve paresis is rare, but facial nerve spasm has been reported more often and it has been suggested that this is probably due to posterior circulation abnormalities induced by the basilar impression. ${ }^{18} 19$ The trigeminal nerve is commonly involved. This is presumably related to the large size and extension of the nerve and its root in the pontomedullary region. Sensory symptoms have been reported in $30 \%-50 \%$ of some series, although motor involvement is rare. ${ }^{10}$ Trigeminal neuralgia (as in case 2) has been reported in several cases and presumably has a vascular aetiology analogous to facial spasm. ${ }^{18}$ It is of interest that Gardner and Dohm, researching the causes of trigeminal neuralgia in elderly women, found a surprisingly high incidence of basilar impression. ${ }^{18}$

All these four cases show radiological evidence of compression in the pontomedullary area. In cases 1 and 2 , the pontine compression has resulted in upper medullary thinning and elongation. The pyramidal tracts project ventrally in the medullary region and so long tract involvement is likely to be common. Lower limbs are almost invariably more affected than upper limbs. De Barros et $a l^{10}$ suggested that pyramidal tract symptoms characterise the basilar impression syndrome whereas vestibulocerebellar symptoms are more typical of tonsillar herniation. ${ }^{10}$ As evidenced in all four cases, the pyramidal signs were the most prominent of the early abnormalities. Bladder disorders are rarely reported in the literature, and none of the four cases has sphincteric symptoms. Pure cerebellar disturbances are rare, although vestibulocerebellar symptoms are common. Case 3 had a spastic ataxia with a heel-shin incoordination, but also a proprioceptive deficit in the toes which deteriorated after operation. There was no evidence of papilloedema in any of the four cases and ventricular enlargement only in case 1 possibly due to compression of the aqueduct. Surprisingly, few cases of basilar impression have documented evidence of raised intracranial pressure. De Barros et $a l^{10}$ found raised intracranial pressure in only $15 \%$ of operated cases and this occurred almost exclusively in those basilar impression cases with tonsillar herniation.

The pathogenesis of neurological symptoms and signs can be attributed to several factors. The most obvious is the direct pressure effect of the clivus, anterior lip of the foramen magnum, body of $\mathrm{C} 1$ and dens on the anterior brainstem leading to early involvement of the pyramidal tracts and subsequently to angulation of the pontomedullary region. Secondly, both operative and postmortem studies in basilar impression have commented on the frequency of arachnoid adhesions and increased vascularisation in the region of the foramen magnum. The adhesions may be due to chronic low grade inflammation related to the interaction of minor trauma from head movements with the abnormal anatomy. The $\mathrm{C} 1$ and $\mathrm{C} 2$ nerve roots are often found to be stretched and traumatised, possibly explaining the frequency 
of occipital pain and headache. Trigeminal neuralgia has reportedly been relieved by removing adhesions from the trigeminal roots. ${ }^{16}$ Finally, vascular abnormalities secondary to mechanical stresses and inflammation are probably important. The transient nature of some of the symptoms also suggest a vascular aetiology. Early angiographic studies of the vertebral system in basilar impression commonly reported an abnormal dorsal displacement of the terminal part of the vertebral artery and the initial segment of the basilar artery. ${ }^{19}$

This is probably due to "kinking" of normal length vessels which have to adapt to a posterior fossa effectively shortened by the process of basilar impression. An abnormal clivus (cases 1 and 2) is likely to accentuate this arching of the vessel. Angiographic studies involving head and neck movements in patients with basilar impression have shown dramatic flow disturbances.

In their discussion of primary basilar impression, Schmidt et al pointed out that for a condition which is congenital, symptoms typically occur quite late, in the third to fifth decades, as opposed to childhood or elderly people. ${ }^{1}$ Likewise, in the previously discussed cases of basilar impression secondary to osteogenesis imperfecta, most develop symptoms in the second to fourth decades, although the range was 3 to 42 years. $^{3620-23}$ The implication is that the maturation process may play some part and that gravity, by virtue of a prolonged upright posture, is an aggravating factor. These possibilities and the relation to osteogenesis imperfecta type are discussed elsewhere. ${ }^{5}$

The true frequency of basilar impression in osteogenesis imperfecta is not known. In a clinic study of 87 patients, Sillence et al detected $25 \%$ of subjects with basilar impression. ${ }^{5}$ Most were asymptomatic. No patients with osteogenesis imperfecta type I had symptoms or abnormal neurological signs. In the group of patients with osteogenesis imperfecta type IV or osteogenesis imperfecta type III/IV - that is, severe sporadic osteogenesis imperfecta, $39 \%$ of patients had symptomatic basilar impression. Basilar impression should be considered in all patients with osteogenesis imperfecta, particularly those in the second to fourth decade with onset of localising symptoms or signs.

Successful surgical treatment of basilar impression by transoral clivectomy with removal of the tip of the dens was carried out on three patients in this series, with marked improvement in postoperative symptoms and signs. All three patients in this series had postoperative velopharyngeal dysfunction with deterioration of speech as reported for a paediatric series with lesions above the foramen magnum. ${ }^{24}$ This can be improved by further pharyngoplasty as recorded in case 1. Craniocervical fusion alone, performed initially in case 1 , as previously reported by Harkey et al may not prevent progress of basilar impression. ${ }^{6}$

Once basilar impression is established, the metrical relation of the dens to the cranial base is not as important as the three dimensional anatomy as reconstructed by MRI. Neurological symptoms and signs give the surest indication of progression. Brain MRI is particularly valuable in defining tonsilar herniation, the earliest development of a syrinx or changes in the posterior fossa which presage the need for intervention. A CT sagittal mid-line reconstruction of the cranial base and hard palate may be useful in the asymptomatic patient as an initial screening to detect those patients with osteogenesis imperfecta who are at risk of progression and require MRI studies.

We thank Associate Professor Robert Ouvrier, neurologist, The Royal Alexandra Hospital for Children Children, Parramatta, Dr Ron Joffe, neurologist, and Dr Ian Farey, orthopaedist, Royal North Shore Hospital and Dr Denis Crimmins, neurologist, Gasford Hospital, and Mr Martin Magee-Collette, neurosurgeon, 3 , and 4 respectively managed by them. We also acknowledge the 3 , and 4 respectively managed by them. We also acknowledge the
assistance of $\mathrm{Mr}$ Alan Crockard, $\mathrm{Mr}$ Alan James, and $\mathrm{Mr}$ Alan assistance of Mr Alan Crockard, Mr Alan James, and Mr Alan
Ransford, Humana Hospital, Wellington, UK, who successfully Ransford, Humana Hospital, Wellington, UK, who successfully
operated on cases 1,2 , and 3 with marked improvement in operated on cases 1,2 , and 3 with marked improvement in
neurological symptoms and signs. We further acknowledge the contribution of Professor TKF Taylor, Department of Orthopaedic and Traumatic Surgery, Royal North Shore Hospital for the management of patient 1 and Mr Michael Besser neurosurgeon, Royal Prince Alfred Hospital, who assessed all patients before the surgical management.

1 Schmidt H, Sartor K, Heckl RW. Bone malformations of the craniocervical region. In: Vinken PJ, Bruyn GW, eds. Handbook of clinical neurology. Amsterdam: North Holland Publishing, 1978;32:1-98.

2 Wald SL, McLaurin RL. Anomalies of the craniocervical junction. In: American Association of Neurologic Surgeons. Pediatric neurosurgery: surgery of the developing nervous system. New York: Grune and Stratton 1982;157-70.

3 Pozo JL, Crockard AH, Ransford AO. Basilar impression in osteogenesis imperfecta. F Bone foint Surg 1984B;66:233-8.

Sillence DO. Osteogenesis Imperfecta. Nosology and genetics. Ann N Y Acad Sci 1987:543:1-25.

5 Sillence DO. Craniocervical abnormalities in osteogenesis imperfecta. Genetic and molecular correlation. Pediatr Radiol 1994;24:427-30.

6 Harkey HL, Crockard HA, Stevens JM, et al. The operative management of basilar impression in osteogenesis impermanagement of basilar impression

7 Dolan KD. Cervicobasilar relationships. Radiol Clin North Am 1977;15:155-66

8 Epstein BS, Epstein JA. The association of cerebellar tonsillar herniation with basilar impression incident to Paget's disease. AfR Am F Roentgenol 1969;107:535

9 Cahan LD, Bentson JR. Considerations in the diagnosis and treatment of syringomyelia and the Chiari malformation. $\mathcal{F}$ Neurosurg 1982;57:24-31.

10 De Barros MC, Ferias W, Ataide L, et al. Basilar impression and Arnold Chiari malformation. A study of 66 cases. $\mathcal{F}$ Neurol Neurosurg Psychiatry 1968;31:596-605.

11 Taylor AR, Chakravorty BC. Clinical syndromes associated with basilar impression. Arch Neurol 1964;10:475-84.

12 Bassi P, Corona C, Contri P, et al. Congenital basilar impression: correlated neurological syndromes. Eur Neurol 1992;32:238-43.

13 Nightingale S, Williams B. Hindbrain hernia headache. Lancet 1987;i:731-4.

14 Sands GH, Newman L, Lipton R. Cough,exertion, and other miscellaneous headaches. Med Clin North Am other miscellane

15 Halmagyi GM. Clinical diagnosis of disordered eye movements. Bull Soc Belge Ophtalmol 1989;237:503-41.

16 Del'osso LF, Daroff RB, Troos BT. Nystagmus and saccadic intrusions and oscillations. In: Duane's clinical ophthalmology. Vol 2. Philadelphia: Lippincott, 1991:1-29.

17 Driesen W. Cited in reference 1. Acta Neurochir (Wien) 1960. 8 Gardner WJ, Dohn D. Trigemminal neuralgia, hemifacial spasm, Paget's disease: significance of this association. Brain 1966;89:555-62.

19 Carella A, Caruso G, Lamberti P. Hemifacial spasm due to elongation and ectasia of the distal segment of the vertebral artery. Report of two cases. Neuroradiology 1973;6:233-6.

20 Hunt TE, Dekabon AS. Modified head-neck support for basilar invagination with brain-stem compression. CMA fournal 1982;126:947-8

21 Rush PJ, Berbrayer D, Reilly BJ. Basilar impression and osteogenesis imperfecta in a three year old girl: CT and osteogenesis imperfecta in a three

22 Brooks ML, Gall C, Wang AM. Osteogenesis imperfecta associated with basilar impression and cerebral atrophy. Comp Med Imaging Graph 1989;13:363-7.

23 Kurimoto $M$, Ohara S, Takaka A. Basilar impression in osteogenesis imperfecta tarda. Case report. $\mathcal{F}$ Neurosurg 1991;74:136-8.

24 Tuite GF, Veres R, Crockard, et al. Pediatric transoral surgery: indications, complications, and long-term outcome. $\mathcal{F}$ Neurosurg 1996;84:573-83. 